\title{
Short Communication Positive thyroid transcription factor I staining strongly correlates with survival of patients with adenocarcinoma of the lung
}

\author{
F Barlési", , D Pinot', A LeGoffic ${ }^{2}$, C Doddoli ${ }^{3}$, B Chetaille ${ }^{2}$, J-P Torre ${ }^{4}$ and P Astoul' \\ 'Faculty of Medicine - Assistance Publique Hôpitaux de Marseille, Department of Thoracic Oncology, Fédération des Maladies Respiratoires, Hôpital \\ Sainte-Marguerite, 13274 Marseille Cedex 09, France; ${ }^{2}$ Faculty of Medicine - Assistance Publique Hôpitaux de Marseille, Department of Pathology, \\ Hôpital Sainte-Marguerite, 13274 Marseille Cedex 09, France; ${ }^{3}$ Assistance Publique Hôpitaux de Marseille, Department of Thoracic Surgery, Hôpital \\ Sainte-Marguerite, 13274 Marseille Cedex 09, France; ${ }^{4}$ Assistance Publique Hôpitaux de Marseille, Department of Medical Information, Hôpital de la \\ Timone, 13285 Marseille Cedex 05, France
}

This study investigated the relation between positive thyroid transcription factor I (TTFI) staining and survival of patients affected by primary adenocarcinoma (ADC) of the lung. Pathological tissue from consecutive ADC patients was collected from 2002 to 2004. The anti-TTFI antibody (8G7G3/I, dilution of I/200) was used. Thyroid transcription factor I staining was assessed for each tumour as positive or negative. Probability of survival was estimated by Kaplan-Meier and difference tested by log-rank test. A Cox's regression multivariate analysis was carried out. In all, 106 patients were studied (66\% male, 69\% PSO-I, 83\% with stage III or IV). Tumours expressed positive TTFI staining in 66\% of cases. Multivariate analysis demonstrated an independent lower risk of death for patients whose tumour expresses positive TTFI staining $(H R=0.5 \mathrm{I}, 95 \% \mathrm{Cl} 0.30-0.85 ; P=0.0 \mathrm{I})$ and higher grade of differentiation $(H R=0.40,95 \% \mathrm{Cl} 0.24-0.68 ; P=0.00 \mathrm{I})$. In conclusion, positive TTFI staining strongly and independently correlates with survival of patients with primary ADC of the lung.

British Journal of Cancer (2005) 93, 450-452. doi: I0.1 038/sj.bjc.66027I7 www.bjcancer.com

Published online 26 July 2005

(c) 2005 Cancer Research UK

Keywords: non-small-cell lung cancer; adenocarcinoma; thyroid transcription factor I; prognosis; differentiation; metastatic stage

Primary adenocarcinoma (ADC) of the lung has risen in incidence, now reaching approximately $50 \%$ of non-small-cell lung cancer (NSCLC). However, identification of primary ADC is sometimes difficult but has been enhanced by the use of the thyroid transcription factor 1 (TTF1) immunostaining. Indeed, positive TTF1 staining has been shown for $75-80 \%$ of primary ADC while negative for virtually all squamous carcinoma (SCC) of the lung as well as for extrapulmonary but thyroid tumours (Stenhouse et al, 2004).

The prognostic value for a positive TTF1 staining has been associated with contradictory results for NSCLC patients. A study on 222 stage I NSCLC patients demonstrated no survival difference associated with TTF1 immunoreactivity (Pelosi et al, 2001), while another study correlated a positive TTF1 staining of resected NSCLC with a poor survival (Puglisi et al, 1999). On the other hand, four studies on early stages of NSCLC associated a positive TTF1 staining with a longer survival (Haque et al, 2002; Myong, 2003; Tan et al, 2003; Saad et al, 2004). However, all these studies were interested in surgical NSCLC patients and all but one (Saad et al, 2004) included both ADC and non-ADC histological types of NSCLC.

The aim of the present study was to investigate the relation of a positive TTF1 staining with survival of patients (1) affected exclusively by a primary ADC of the lung and (2) mainly presenting with locally advanced or metastatic stage of the disease.

*Correspondence: Dr F Barlési; E-mail: fabrice.barlesi@mail.ap-hm.fr Revised 21 April 2005; accepted 20 June 2005; published online 26 July 2005

\section{PATIENTS AND METHODS}

All consecutive patients diagnosed with ADC from January 2002 to May 2004 at our department were included in the study. Histological subclassification was carried out according to the World Health Organization classification (Travis et al, 1999). Performance status (PS) was estimated using the Eastern Cooperative Oncology Group (ECOG) scale. Clinical examination and computed tomographic scan of the chest, abdomen and brain were carried out systematically.

Pathological tissue was obtained from each patient before treatment. The pathological tissue was obtained either from the primary tumour in the majority of cases, or from mediastinal lymph nodes $(n=10)$ or distant metastases $(n=5)$. The pathological tissue was extracted from surgical specimens when available. Tumours have been classified into poorly, moderately and welldifferentiated tumours (Travis et al, 1999). Immunohistochemical staining was carried out on formalin-fixed, paraffin-embedded tissue samples, using a standard streptavidin-biotin-based method. The anti-TTF1 antibody (8G7G3/1, mouse monoclonal antibody; Dako, Ely, Cambridgeshire, UK) was used at a dilution of $1 / 200$. Scoring was carried out independently by two pathologists (AL and BC). For each tumour, neoplastic cells were assessed as positive or negative for TTF1 staining (Figure 1). Sole nuclear staining was considered as a positive result. Any positive nuclear staining was sufficient to deem a tumour as positive for TTF1 staining. In each case, normal alveolar walls served as positive internal control. All patients consented to treatment and were treated with thoracic surgery, radiation therapy and/or 
chemotherapy, in accordance with national and international guidelines (Depierre et al, 2003) after a multidisciplinary assessment of their disease.

Survival data were updated in March 2005. One patient was lost to follow-up. Probability of survival was estimated using the Kaplan-Meier method. Differences between survival were tested by means of log-rank test. A multivariate regression analysis was carried out with Cox's regression using the forward maximum

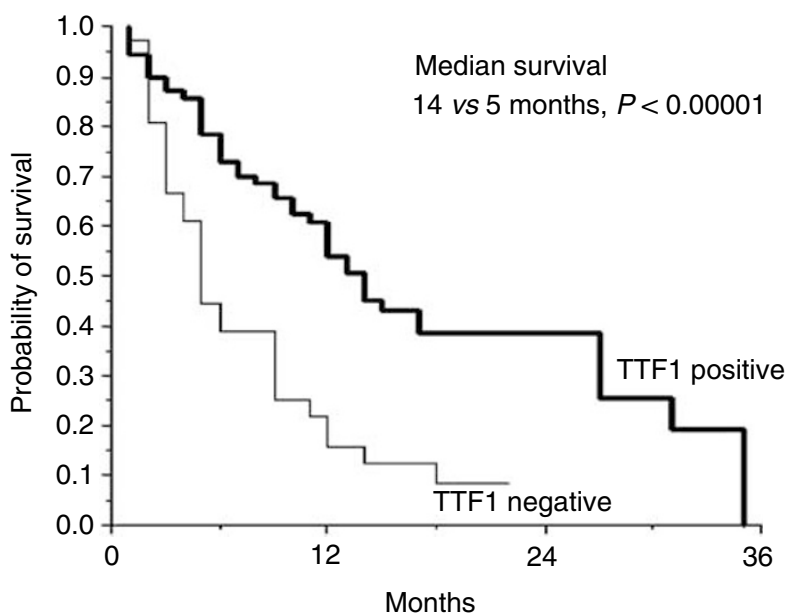

Figure I Survival curves of patients whose tumours showed positive and negative TTFI staining.
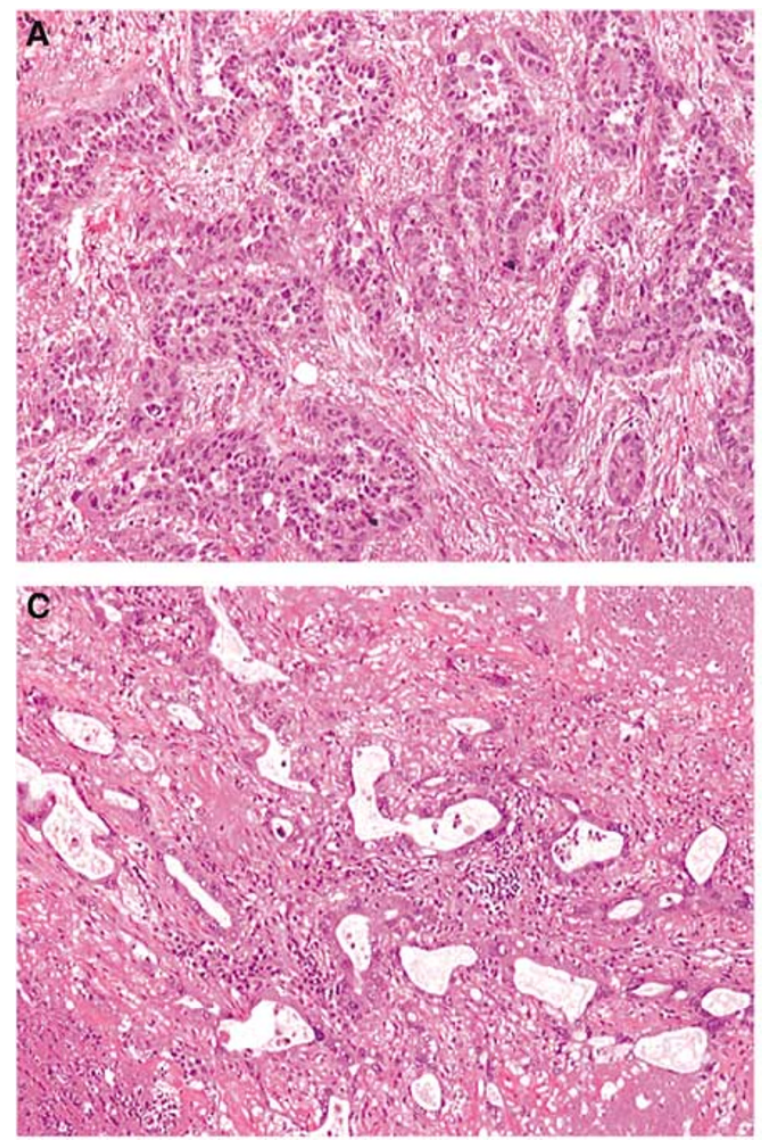

likelihood method. All variables with a $P$-value less than 0.20 at the time of univariate analysis were entered into the model. A $P$-value less than 0.05 was considered as significant.

\section{RESULTS}

In all, 106 patients were included into the study (Table 1). Tumours expressing positive TTF1 staining were associated neither with demographics (i.e. gender and age) nor with disease characteristics (i.e. PS, TNM stage or presence of metastasis).

Table I Main clinical and pathological characteristics of the 106 patients

\begin{tabular}{ll}
\hline & \multicolumn{1}{c}{$\boldsymbol{n}(\%)$} \\
\hline Age, $<70 / \geqslant 70$ years & $82(77) / 24(23)$ \\
Gender, women/men & $36(34) / 70(66)$ \\
PS, $0-1 / \geqslant 2$ (ECOG) & $73(69) / 33(31)$ \\
Stage & \\
$\quad$ Early stage (I and II) & $18(17)$ \\
Locally advanced stage (IIIA and IIIB) & $35(33)$ \\
Distant metastasis (IV) & $53(50)$ \\
Histological differentiation & $38(36)$ \\
$\quad$ Poorly differentiated & $47(44)$ \\
Moderately differentiated & $21(20)$ \\
$\quad$ Well differentiated & $70(66) / 36(33)$ \\
TTFI staining (positive/negative) &
\end{tabular}

aUICC Classification. PS = performance status; TTFI = thyroid transcription factor 1 .
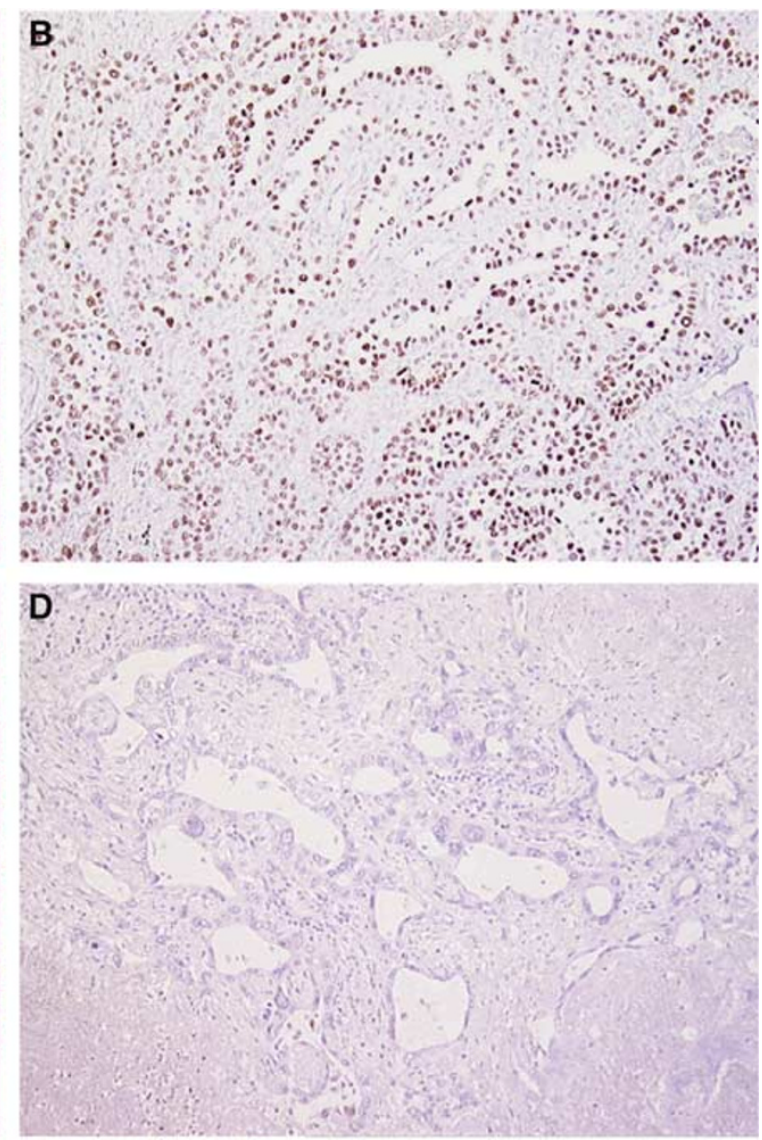

Figure 2 Acinar ADC (haematoxylin and eosin, $\times 100)(\mathbf{A})$ showing positive nuclear TTFI staining (B), and acinar ADC (haematoxylin and eosin, $\times$ 100) $(\mathbf{C})$ showing negative TTFI staining $(\mathbf{D})$. 
Table 2 Major results of univariate analysis

\begin{tabular}{lcc}
\hline & Median survival (months) & $P$ \\
\hline PS & 12 & NS \\
O or I & 5 & \\
$\geqslant 2$ & 10 & NS \\
Stage & 12 & \\
I and II & 7 & \\
IIIA and IIIB & & \\
IV & 14 & \\
TTFI staining & 5 & \\
Positive & & \\
Negative & 5 & \\
Differentiation & 13 & \\
Poorly & 12 & \\
Moderately & & \\
Well & &
\end{tabular}

PS = performance status (ECOG); TTFI = thyroid transcription factor I; NS = nonsignificant.

Thyroid transcription factor 1 staining was not associated with tumours differentiation, as poorly, moderately and well-differentiated tumours expressed a positive TTF1 staining in $21(30 \%$ of the tumours showing positive TTF1 staining), 35 (50\%) and 14 (20\%) cases, respectively $(P=0.177)$.

At the time of analysis, 75 patients were deceased. In univariate analysis, tumours differentiation and TTF1 staining significantly correlated with survival. Tumours stage as well as PS also influenced survival, without reaching statistical significance (Table 2). Multivariate analysis demonstrated a statistically significant and independent lower risk of death for patients whose tumour expresses a positive TTF1 staining ( $\mathrm{HR}=0.40,95 \% \mathrm{CI}$ $0.25-0.65 ; P<0.0001$ ) (Figure 2 ), and a statistically significant and independent lower risk of death for patients whose tumour expresses higher grade of differentiation $(\mathrm{HR}=0.64,95 \% \mathrm{CI} 0.46-$ $0.91 ; P=0.01)$.

\section{REFERENCES}

Depierre A, Lagrange JL, Theobald S, Astoul P, Baldeyrou P, Bardet E, Bazelly B, Brechot JM, Breton JL, Douillard JY, Grivaux M, Jacoulet P, Khalil A, Lemarie E, Martinet Y, Massard G, Milleron B, Molina T, Moro-Sibilot D, Paesmans M, Pujol JL, Quoix E, Ranfaing E, Riviere A, Sancho-Garnier H, Souquet PJ, Spaeth D, Stoebner-Delbarre A, Thiberville L, Touboul E, Vaylet F, Vergnon JM, Westeel V, FNCLCC (2003) Summary report of the standards, options and recommendations for the management of patients with non-small-cell lung carcinoma (2000). Br J Cancer 89(Suppl. 1): S35-S49

Haque AK, Syed S, Lele SM, Freeman DH, Adegboyega PA (2002) Immunohistochemical study of thyroid transcription factor-1 and HER2/neu in non-small cell lung cancer: strong thyroid transcription factor-1 expression predicts better survival. Appl Immunohistochem Mol Morphol 10: 103 - 109

Myong NH (2003) Thyroid transcription factor-1 (TTF-1) expression in human lung carcinomas: its prognostic implication and relationship with expressions of $\mathrm{p} 53$ and Ki-67 proteins. J Korean Med Sci 18: 494-500

Pelosi G, Fraggetta F, Pasini F, Maisonneuve P, Sonzogni A, Iannucci A, Terzi A, Bresaola E, Valduga F, Lupo C, Viale G (2001) Immunoreactivity for thyroid transcription factor-1 in stage I non-small cell carcinomas of the lung. Am J Surg Pathol 25: 363-372

\section{DISCUSSION}

This study highlights the decreased risk of death for patients with primary ADC of the lung showing a positive TTF1 staining $(\mathrm{HR}=0.40,95 \%$ CI $0.25-0.65 ; P<0.0001)$.

Previous studies suggested either a favourable or an unfavourable prognosis for patients with tumour showing a positive TTF1 staining. However, all these studies but one (Saad et al, 2004) included NSCLC patients with a mixture of histological types. However, the prevalence of a positive TTF1 staining varies throughout histological types, from $80 \%$ for ADC to less than $10 \%$ for SCC. This heterogeneity probably explains the conflicting results previously reported. In addition, available data on the prognosis associated with TTF1 staining considered exclusively surgically treated NSCLC patients. Thus, the results reported herein extend the favourable prognosis related to a positive TTF1 staining to patients with advanced stage of primary ADC of the lung.

The reasons why TTF1 is related to prognosis of patients with primary ADC of the lung are unclear. A positive TTF1 staining has been inversely related to the proliferative activity evaluated through Ki-67 expression, usually considered as a marker of poor prognosis in NSCLC (Pelosi et al, 2001; Myong, 2003). On the other hand, a correlation between positive TTF1 staining and various molecular markers expression such as p53 or HER2/neu involved in lung carcinogenesis has been suggested (Haque et al, 2002; Yatabe et al, 2002; Myong, 2003). Thus, further biological investigations are needed.

Finally, a significant relationship between positive TTF1 staining and female gender or nonsmoker status has been suggested (Yatabe et al, 2002). Thus, a correlation with epidermal growth factor receptor tyrosine kinase inhibitors activity should be prospectively investigated. In fact, a TTF1 influence on patients' survival through difference in treatment sensibility might be hypothesised.

In conclusion, positive TTF1 staining strongly and independently correlates with survival of patients with ADC of the lung by a way to be identified.

Puglisi F, Barbone F, Damante G, Bruckbauer M, Di Lauro V, Beltrami CA, Di Loreto C (1999) Prognostic value of thyroid transcription factor-1 in primary, resected, non-small cell lung carcinoma. Mod Pathol 12: $318-324$

Saad RS, Liu YL, Han H, Landreneau RJ, Silverman JF (2004) Prognostic significance of thyroid transcription factor-1 expression in both earlystage conventional adenocarcinoma and bronchioloalveolar carcinoma of the lung. Hum Pathol 35: 3-7

Stenhouse G, Fyfe N, King G, Chapman A, Kerr KM (2004) Thyroid transcription factor 1 in pulmonary adenocarcinoma. J Clin Pathol 57: 383-387

Tan D, Li Q, Deeb G, Ramnath N, Slocum HK, Brooks J, Cheney R, Wiseman S, Anderson T, Loewen G (2003) Thyroid transcription factor-1 expression prevalence and its clinical implications in non-small cell lung cancer: a high-throughput tissue microarray and immunohistochemistry study. Hum Pathol 34: 597-604

Travis WD, Colby TV, Corrin B, Shimosato Y, Brambilla E, in collaboration with pathologists from 14 countries (1999) World Health Organization International Histological Classification of Tumours. Histological Typing of Lung and Pleural Tumours 3rd edn. Berling: Springer-Verlag

Yatabe Y, Mitsudomi T, Takahashi T (2002) TTF-1 expression in pulmonary adenocarcinomas. Am J Surg Pathol 26: 767-773 